\title{
Ovos de Trichuris vulpis em amostras fecais de cães analisadas na região de Pelotas, RS, entre 2015 e 2018
}

\author{
Trichuris vulpis eggs in stool samples of dogs analyzed in the region of \\ Pelotas, RS, between 2015 and 2018
}

\begin{abstract}
Alexsander Ferraz (D)*, Bruna dos Santos Pires (D), Eugênia Tavares Barwaldt (D), Eduarda Machado dos Santos (D), Paola Renata Joanol Dallmann (D), Tanize Angonesi de Castro (D), Márcia de Oliveira Nobre (D), Leandro Quintana Nizoli iD
\end{abstract}

Universidade Federal de Pelotas (UFPel), Pelotas, RS, Brasil

\section{Resumo}

Trichuris vulpis é um helminto que vive no intestino de cães, principalmente no ceco e no colon. Cães infectados por este parasito eventualmente podem apresentar diarreia, às vezes sanguinolenta, vômito e dor abdominal. Os ovos deste parasito podem permanecer viáveis no ambiente por vários anos, sendo fonte de infecção para seu hospedeiro definitivo. Este trabalho teve como objetivo demonstrar a frequência de T. vulpis em amostras fecais de cães, recebidas no Laboratório de Doenças Parasitárias (Ladopar) da Universidade Federal de Pelotas (UFPel) entre os anos de 2015 e 2018. As amostras foram processadas pela técnica de WillisMollay (Willis, 1921), que utiliza o princípio da flutuação em solução hipersaturada para pesquisa de ovos leves de helmintos. Das 1356 amostras recebidas, 217 (16\%) foram positivas para este gênero, sendo que o ano de 2016 foi o que apresentou maior frequência (20\%).

Palavras-chave: Fezes. Helminto. Coproparasitologia.

\begin{abstract}
Trichuris vulpis is a helminth that lives in the intestines of dogs, mainly in the cecum and in the colon, and dogs infected by this parasite can present diarrhea, sometimes bloody, vomiting and abdominal pain. The eggs of this parasite can remain viable in the environment for several years, being a source of infection for its definitive host. This study aimed to demonstrate the frequency of T. vulpis
\end{abstract}


in fecal dog samples received at the Parasitic Diseases Laboratory (Ladopar) of the Universidade Federal de Pelotas (UFPel) between 2015 and 2018. The samples were processed by the technique of Willis-Mollay (Willis, 1921), which uses the principle of fluctuation in hypersaturated solution for the search of light helminth eggs. Of the 1356 samples received, 217 (16\%) were positive for this genus, and the year of 2016 was the one with the highest frequency (20\%).

Keywords: Feces. Helminth. Coproparasitology.

\section{Introdução}

Trichuris vulpis é um nemátodeo que tem como hospedeiros definitivos o cão, o gato e canídeos selvagens, sendo encontrado no ceco e no cólon destes animais (Taylor et al., 2007), onde realiza a histio e hematofagia (Prats et al., 2005). A transmissão ocorre por via fecal oral, através da ingestão de ovos contendo as larvas infetantes (L1), presentes na água ou no solo, contaminadas pelas fezes de animais parasitados (Zajac e Conboy, 2012).

Os ovos de T. vulpis são bastante resistentes, podendo, em condições ideais, permanecer viáveis no solo por longo período, apresentando uma longevidade de até três a quatro anos no ambiente (Trillo-Altamiro et al., 2003).

$\mathrm{Na}$ maioria das vezes, as infecções são assintomáticas ou leves, sendo que os principais sinais clínicos apresentados, principalmente em animais com elevada carga parasitária, são: diarreia, às vezes com sangue, vômito, dor e distensão abdominal; alguns cães podem apresentar prolapso retal (Longo et al., 2008). Já houve registro de casos de T. vulpis como agente causador de infecção entérica no homem (Coura, 2008).

Pelo fato de os sinais clínicos não serem característicos, sendo comuns a outras doenças, o diagnóstico definitivo pode ser obtido através de técnicas coproparasitológicas de flutuação (Foreyt, 2002; Fortes, 2004), onde é possível visualizar os ovos, que apresentam como características formato de limão, coloração acastanhada e possuem dois opérculos nas extremidades (Urquhart et al., 1998).
O tratamento consiste na administração de antiparasitários. Febendazol, moxidectina, milbemicina e a associação de emodepside com praziquantel, e de pirantel com febantel e praziquantel costumam ser eficazes (Alho et al., 2010).

Embora não muito relatado, alguns estudos indicam seu potencial zoonótico, como Sakano et al. (1980), Masuda et al. (1987), Singh et al. (1993) e Mirdha et al. (1998), que relataram a ocorrência de larva migrans visceral causada por T. vulpis, além de úlceras e infecções intestinais e pulmonares causadas pelo parasita. Dunn et al. (2002) relataram um caso humano de T. vulpis em uma mulher com úlcera duodenal e diarréia crônica, que convivia com cinco cães, o que evidencia o seu possível potencial zoonótico.

Este trabalho teve como objetivo demonstrar a frequência de ovos de Trichuris vulpis em amostras fecais de cães, recebidas no Laboratório de Doenças Parasitárias da Universidade Federal de Pelotas (UFPel) entre os anos de 2015 e 2018.

\section{Material e métodos}

Foram recebidas e analisadas no Laboratório de Doenças Parasitárias (Ladopar) da Faculdade de Veterinária (FAVet) da Universidade Federal de Pelotas (UFPel), entre os anos de 2015 e 2018, 1356 amostras fecais de cães. O material recebido foi acondicionado em pote plástico e mantido sob refrigeração $\left(5^{\circ} \mathrm{C}\right)$ até o momento da análise, que era realizada em até 24 hs.

A técnica utilizada para processamento das amostras foi a de Willis-Mollay (Willis, 1921), que utiliza o princípio da flutuação em solução hipersaturada para a pesquisa de ovos leves de helmintos e oocistos de protozoários. A leitura das lâminas foi realizada em microscópio óptico, com aumento de 100x.

\section{Resultados e discussão}

Das amostras analisadas, 217 (16\%) foram positivas para ovos de Trichuris vulpis, sendo que o ano de 2016 foi o que apresentou maior frequência de amostras positivas (20\%) (Tabela 1). 
Tabela 1 - Frequência de ovos de Trichuris vulpis em amostras fecais de cães processadas no Laboratório de Doenças Parasitárias da UFPel entre os anos 2015 e 2018

\begin{tabular}{|c|c|c|c|c|c|}
\hline \multirow{2}{*}{ Amostras } & \multicolumn{4}{|c|}{ Ano } & \multirow{2}{*}{ Total } \\
\hline & 2015 & 2016 & 2017 & 2018 & \\
\hline Total (n) & 204 & 505 & 449 & 198 & 1356 \\
\hline Positivas (n) & 38 & 101 & 46 & 32 & 217 \\
\hline Média (\%) & 18,6 & 20,0 & 10,2 & 16,2 & 16,0 \\
\hline
\end{tabular}

Das 1356 amostras, $16 \%$ continham ovos de $T$. vulpis, evidenciando parasitismo mais elevado do que o encontrado por Farias et al. (2013) e Leal et al. (2015) que, ao avaliarem cães domiciliados, obtiveram apenas um animal parasitado por Trichuris vulpis, correspondendo a 0,9\% (1/107) e $0,45 \%(1 / 221)$, respectivamente. Já Vital et al. (2012) não encontraram ovos do parasito em seu estudo, realizado no Distrito Federal.

Silva et al. (2007), entretanto, observaram ovos do nematódeo em 11,25\% das 240 amostras analisadas de cães domiciliados em um bairro de Santa Maria, RS, e Stalliviere et al. (2013) em 8\% $(42 / 523)$ dos cães analisados em Lages, SC. Do mesmo modo, Prates et al. (2009) encontraram prevalência de 6,2\% (5/81) em cães da periferia de Maringá, PR, e Leite et al. (2004), 3,3\% em Curitiba, PR. No Rio de Janeiro, RJ, cães institucionalizados apresentaram 2,5\% (5/204) de infecção, de acordo com Vasconcellos et al. (2006), e cães errantes da cidade de Goiânia, GO, avaliados por Oliveira et al. (2009), evidenciaram prevalência de 1\% (2/201).

Amostras fecais ambientais também foram utilizadas em outros estudos, as quais continham ovos de T. vulpis, como as coletadas em praias, onde Blazius et al. (2006) verificaram 13,9\% de contaminação em Laguna, SC, e Leite et al. (2006), 2\% em Meia-Praia, Itapema, SC. Alves et al. (2014) encontraram um percentual de 3,1\% de fezes contaminadas em praças públicas em Pindamonhangaba, SP.

O fato de ovos de T. vulpis, em condições ideais de temperatura e umidade, serem bastante resistentes no ambiente, podendo permanecer viáveis por três a quatro anos (Trillo-Altamiro et al., 2003), mostra que este parasito é bastante prevalente em ambientes quentes e úmidos (Fortes,
2004). Estes fatores podem justificar a elevada frequência encontrada no presente estudo (16\%), pois Pelotas, RS é um município caracterizado por apresentar elevada umidade, com média anual de cerca de 80\% (Bretanha e Kobiyama, 2016).

\section{Conclusão}

A partir dos resultados encontrados, concluise que há uma elevada frequência de Trichuris vulpis parasitando cães do município de Pelotas, RS. O significativo número de amostras positivas encontradas neste estudo merece ser destacado, pois devido ao fato de os ovos permanecerem viáveis por longos períodos no ambiente e apresentarem risco zoonótico, medidas devem ser adotadas com o intuito de reduzir a infecção de animais e humanos por este parasito.

\section{Referências}

Alho AM, Seixas R, Rafael T, Carvalho LM. Formas larvares dos helmintos: o elo mais forte na desparasitação do cão e do gato. Rev Vet Med. 2010;12(71):33-46.

Alves APSM, Coelho FAS, Coelho MDG. Frequência de enteroparasitos em fezes de cães coletadas em praças públicas do município de Pindamonhangaba, SP, Brasil. Rev Patol Trop. 2014;43(3):341-50.

Blazius RD, Silva OS, Kauling AL, Rodrigues DFP, Lima MDC. Contaminação da areia do Balneário de Laguna, SC, por Ancylostoma spp., e Toxocara spp. em amostras fecais de cães e gatos. ACM Arq Catarin Med. 2006;35(3):55-8.

Bretanha SSF, Kobiyama M. Estudo do clima no município de Pelotas, RS. Rev Geonorte. 2016;7(27):30-49.

Coura JR. Síntese de Doenças infecciosas e parasitárias. Rio de Janeiro: Guanabara Koogan; 2008. 322 p.

Dunn JJ, Columbus ST, Aldeen WE, Davis M, Carroll KC. Trichuris vulpis recovered from a patient with chronic diarrhea and five dogs. J Clin Microbiol. 2002;40(7):2703-4. 
Farias ANS, Silva M, Oliveira JBS, Rocha LB, Santos KR. Diagnóstico de parasitos gastrointestinais em cães do município de Bom Jesus, Piauí. Rev Acad Ciênc Agrar Ambient. 2013;11(4):431-5.

Foreyt WJ. Veterinary Parasitology: reference manual. 5 ed. Ames, lowa: lowa State University Press; 2002. 284 p.

Fortes E. Parasitologia Veterinária. 4 ed. São Paulo: Ícone; 2004. 608 p.

Leal PDS, Moraes MIMR, Barbosa LLO, Figueiredo LP, Lima e Silva S, Lopes CWG. Parasitos gastrintestinais em cães domiciliados atendidos em serviço de saúde animal, Rio de Janeiro, Brasil. Rev Bras Med Vet. 2015;37(Supl.1):37-44.

Leite LC, Bandeira CR, Cirio SM, Luz E, Diniz JMF, Leite SC, et al. Ocorrência de ovos de Ancylostoma spp. e Trichuris spp. em fezes de cães em Meia-Praia, Itapema, Santa Catarina, Brasil. Estud Biol. 2006;28(65):105-10.

Leite LC, Marinoni LP, Círio SM, Diniz JMF, Silva MAN,

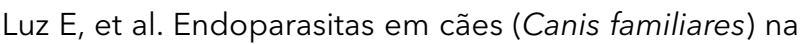
cidade de Curitiba - Paraná - Brasil. Arch Vet Sci. 2004; 9(2):95-9.

Longo CEM, Santos GR, Oliveira JLS, Neves MF. Trichuris Vulpis. Rev Cient Eletr Med Vet. 2008;6(11):1-6.

Masuda $Y$, Kishimoto T, Ito $H$, Tsuji M. Visceral larva migrans caused by Trichuris vulpis presenting as a pulmonary mass. Thorax. 1987;42(12):990-1.

Mirdha BR, Singh IG, Samantray JC, Mishra B. Trichuris vulpis infection in slum children. Indian J Gastroenterol. 1998; 17(4):154.

Oliveira VSF, Melo DPG, Fernandes PR, Schulze CMB, Guimarães MS, Silva AC. Ocorrência de helmintos gastrintestinais em cães errantes na cidade de Goiânia Goiás. Rev Patol Trop. 2009;38(4):279-83.

Prats A, García F, Dumon C, Martí S, Coll V. Neonatologia e pediatria canina e felina. São Paulo: Interbook; 2005. 469 p.

Prates L, Pacheco LS, Kuhl JB, Dias MLGG, Araújo SM, Pupulin ART. Frequência de parasitos intestinais em cães domiciliados da cidade de Maringá, PR. Arq Bras Med Vet Zootec. 2009;61(6):1468-70.

Sakano T, Hamamoto K, Kobayashi Y, Sakata Y, Tsuji M, Usui T. Visceral larva migrans caused by Trichuris vulpis. Arch Dis Child. 1980;55(8):631-3.

Silva AS, Ceolin LV, Cargnelutti JF, Pessoa GA, Oliveira CB, Quintal APN, et al. Prevalência de parasitismo em cães domiciliados num bairro de Santa Maria - RS. Saude. 2007;33(1):27-31.

Singh S, Samantary JC, Singh N, Das GB, Verma IC. Trichuris vulpis infection in an Indian tribal population. J Parasitol. 1993;79(3):457-8.

Stalliviere FM, Dalla Rosa L, Bellato V, Souza AP, Sartor AA, Moura AB. Helmintos intestinais em cães domiciliados e aspectos socioeconômicos e culturais das famílias proprietárias dos animais de Lages, SC, Brasil. Arch Vet Sci. 2013;18(3):22-7.

Taylor MA, Coop RL, Wall RL. Veterinary Parasitology. 3 ed. Hoboken, NJ, USA: Willey-Blackwell; 2007.600 p.

Trillo-Altamirano MP, Carrasco AJ, Cabrera R. Prevalência de helmintos enteroparásitos zoonóticos y factores em Canis familiaris em uma zona urbana de la ciudad de Ica, Peru. Parasitol Latinoam. 2003;58(3-4):136-41.

Urquhart GM, Armour J, Duncan JL, Dunn AM, Jennings FW. Parasitologia Veterinária. 2 ed. São Paulo: Guanabara Koogan; 1998.

Vasconcellos MC, Barros JSL, Oliveira CS. Parasitas gastrointestinais em cães institucionalizados no Rio de Janeiro, RJ. Rev Saude Publica . 2006;40(2):321-3.

Vital TE, Barbosa MRA, Alves DSMM. Ocorrência de parasitos com potencial zoonótico em fezes de cães e gatos do Distrito Federal. Ensaios e Cienc. 2012;16(1):9-23.

Zajac AM, Conboy GA. Veterinay Clinical Parasitology. 8 ed. Chichester, WS, UK: Willey-Blackwell; 2012.

Willis $\mathrm{HH}$. A simple levitation method for the detection of hookworm ova. Med J Aust. 1921;2(18):375-6. 